\title{
Improving Encryption Digital Watermark by Using Blue Monkey Algorithm
}

\author{
OMAR YOUNIS ABDULHAMMED \\ College of Science, Department of Computer Science, University of Garmian, Kalar, Iraq \\ (e-mail: Omar.y@garmian.edu.krd, Omaralaa78@yahoo.com) \\ Corresponding author: Omar Younis Abdulhammed (e-mail: Omar.y@garmian.edu.krd).
}

\begin{abstract}
$:$ ABSTRACT Watermarking enables the users to share the digital contents in public domain without any issue. In the present day, the tremendous development in the digital technologies and networks caused an increase in the threats of unauthorized copying, tampering of digital media and image theft. To face these threats, digital watermark technology can be applied. However, the current paper uses new technique with two main algorithms that are the following ones: improved honey algorithm that is used to encrypt the digital watermark and blue monkey meta-heuristic algorithm which is used to find the best location in the host image to hide the digital watermark. Furthermore, in order to check the security and the robustness of the proposed method against various common image processing attacks such as Gaussian noise, Rotation, Salt and pepper noise, Sharpen, Median filter, Average filter, compression and Cropping is computed, certain performance metrics such as Peak to Signal Noise Ratio (PSNR)and Mean Square Error (MSE) also are computed. Likewise, Normalized Correlation (NC) is used to check similarity between the original and extracted digital watermark. The results demonstrate that the proposed method is efficient, provides high security and robustness against most attacks compared to the pervious methods.
\end{abstract}

: KEYWORDS Digital media, watermark, blue monkey, meta-heuristic, honey encryption, host image.

\section{INTRODUCTION}

$\mathrm{T}$ HE development of image processing algorithm, application programs and network technologies has fascinated the process of changing, reproducing, duplicating digital images at low cost and approximately immediate delivery without any degradation of quality, therefore these threats and challenges must be faced, one of the effective solutions to meet these threats is watermarking [1]. Digital image watermarking is a method in which the watermark is merged with the object to be protected and then extracted from it. These methods ensure tamper-resistance, authentication, content verification, and integration of the image [2]. It is not simple to remove a watermark by displaying or converting the watermarked data into other file formats. Therefore, after an attack, it is possible to obtain information about the transformation from the watermark [3]. Digital-to-analog conversion, compression, file format changes, re-encryption, and decryption can also be survived through digital image watermarking techniques [4].

\section{DIGITAL WATERMARKING}

Digital watermarking is a number of bits embedded into file that identifies the file's copyright information, file like audio, image, or video data [5]. This technique is widely used in copyright protection and content authentication of images in multimedia [6], it is considered more secure when compared to effectively existing strategies of sending the interactive media information safely. Watermarks are so named because of their corresponding with the impact of water droplets spread over the sheet of paper. Digital image watermarking is essentially a section of applied science that reviews the digital images and their changes so as to promote their quality or to extract data [7]. Fig. 1 shows the stages in image watermarking [8]. 


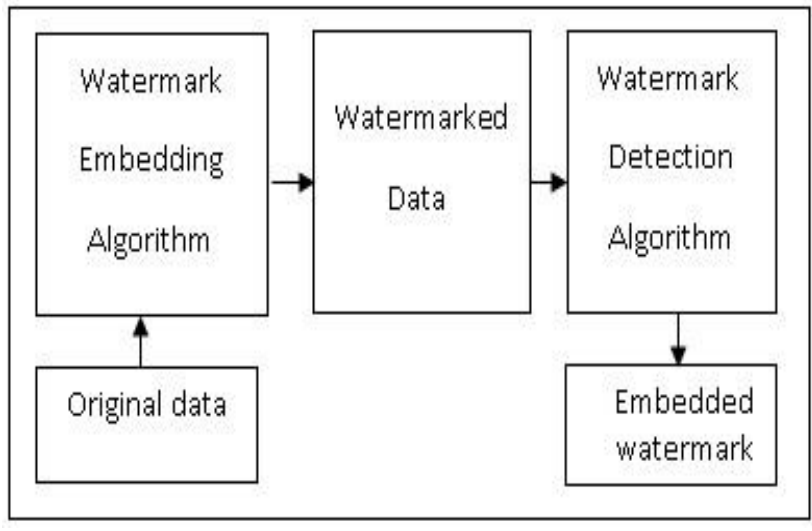

Figure 1. Stages of Watermarking

\section{HONEY ENCRYPTION}

Honey Encryption (HE) scheme was first introduced and used by Juels and Ristenpart in 2014 to protect the password based encryption of RSA secret keys and credit card numbers [9]. The DTE (Distributed Transforming Encoding) is the main idea behind pure honey encryption technique. Honey encryption manages the space of plaintext via DTE. Let the probability distribution over the message space be $\mathrm{p}$ over the message L. The distribution transforming encodes the message $\mathrm{L}$ as a $\mathrm{K}$ bit seed $\epsilon\{0,1\} \mathrm{K}$ and decodes the message by inverse DTE method, decode (S) $=$ L. DTE is a good model of the message distribution. The internal structure of the HE includes DTE encryption and DTE decryption. The two algorithms describe the net functioning of the Honey Encryption. Algorithm (1) and (2) show the steps of honey encryption and decryption respectively

\section{Algorithm 1. Honey encryption algorithm}

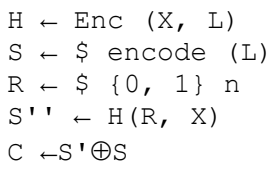

\section{Algorithm 2. Honey decryption algorithm}

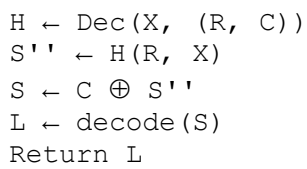

$\mathrm{H}$ is a cryptographic hash function, $\mathrm{X}$ is a key, $\mathrm{L}$ is a message, $\mathrm{S}$ is a seed, $\mathrm{R}$ is a random string, $\mathrm{C}$ is a cipher text and $\leftarrow \$$ indicates that Honey Encryption algorithm may use some number of uniform random bits. When the Honey Encryption is applied to the plaintext message L, it first encodes the message $\mathrm{L}$ to $\mathrm{S}$ and then encrypts $\mathrm{S}$ by a key $\mathrm{X}$ using suitable symmetric encryption algorithm. The above algorithms describe these steps clearly, high message recovery security is provided by Honey encryption [10].

\section{BLUE MONKEY ALGORITHM}

Is algorithmic program mimics behavior of the Blue Monkey. To model such interactions, every cluster of monkey's area unit is needed to maneuver over the search area. Referring to the previous argument, when monkeys are divided into teams, the monkey that begins to search for food at long distances area and the stronger one is not among the scope of traditional vision. In addition, the male Cercopithecus mitis has either little or no interaction with the young ones and because of the territorial nature of the Cercopithecus mitis, the young males should go out as earlier to become more successful so they challenge the dominant male of another family in this point. In case one male succeeded to defeat another male, he becomes the leader of that family and he can offer food supplies, place to live and socialization for young males [11]. The new position of each blue monkey in the group depends on how good the blue monkey's position in that group is. However, this behavior is delineating by the following equations:

$$
\begin{gathered}
\text { Rate } \left._{(\mathrm{i}+1)}=\left(0.7 * \text { Rate }_{\mathrm{i}}\right)+\left(\mathrm{W}_{\text {leader }}-\mathrm{W}_{\mathrm{i}}\right) * \text { rand } \mathrm{W}_{\text {best }}-\mathrm{X}_{\mathrm{i}}\right) \\
\mathrm{Xi}+1=\mathrm{Xi}+\text { Ratei }+1 * \text { rand }
\end{gathered}
$$

where, Rate represents the monkey power rate, $\mathrm{W}_{\text {leader }}$ is the leader weight, $\mathrm{Wi}$ is the monkey weight at which all weights are random numbers between $[4,6], X$ is the monkey position, $\mathrm{X}_{\text {best }}$ is the leader position and rand is an arbitrary number between $[0,1]$, [12].

\section{LITERATURE REVIEW}

This section presents some of the previous methods in the field of watermarking methods. In Paper [13] an effective watermark method has been proposed that is used to preserve the patient's medical image and information which is sent over the internet. This method depends on combining two techniques where first technique uses a reversible watermarking by merging the LSB \& cryptographies tools and second technique uses CDMADWT domain. The result shows that the method is very robust against different attacks like salt and pepper noise. In paper [14] two techniques are used which are data hiding and ciphering, where the watermark is encrypted by using XOR operation and then embedded in the DWT domain, the results proved that the method is robust against many attacks.

Paper [15] proposed a new method consisting of mainly two techniques, the first is discrete wavelet transform (DWT) and the second is least significant bit (LSB), the concealment process was done in three colors (RGB). The results proved the efficiency of the proposed method against attacks.

In paper [16] a new robust digital watermarking algorithm for embedding content into the DCT was presented. The watermark is embedded in low and midfrequency of DCT components. The experimental results illustrated perfect apparent imperceptibility and resiliency of the proposed scheme versus several attacks, such as geometric, JPEG compression, noise, filtering and enhancement attacks. In paper [17] a robust watermarking 
algorithm depending on DCT for securing Iris images was proposed. Because the security of biometric trait is very important, a robust security must be provided. So, this method is used for protecting the integrity of the iris images using a demographic text as a watermark.

The watermark text is embedded in the middle band frequency region of the iris image (in the middle band coefficients pairs of the DCT). The experimental results showed that watermark is robust against several attacks. Also, the results showed that the method did not offer discernible decrease on iris image quality or biometric recognition performance.

In paper [18] a new scheme known as progressive secret sharing was proposed. This scheme consists of two various processes depending on secret image that is transformed for a group of shared images. The first process uses the public grids that are random. Further the second process uses mainly the exclusive-OR (XOR) operation whose purpose is to generate a set of shared images.

Paper [19] proposed an algorithm depending on digital image watermarking that is based upon entropy of the blocks along with the histogram. The method includes splitting the cover image into a number of blocks and then these blocks are selected depending on the base of entropy values for the purpose of hiding a watermark. Then the watermark is concealed within the selected blocks using the histogram shape approach.

All previous papers did not depend on ideas found in nature to solve its problem through using meta-heuristic algorithm also no improved encryption algorithm was used to increase the security.

\section{PROPOSED METHOD}

As long as the watermarks provide a solution to all copyright problems, unauthorized use and illegal usage, the proposed method is characterized by the following points:

- Using BMA to find the best positions in the host image with intelligent way through relying on the fitness values of these positions, which is used to conceal the digital watermark, and that will be difficult to detect by attacker

- Using improved honey encryption to cipher the digital watermark image before concealing it through using the address values of the locations chosen by BMA as keys, that will increase the security.

- Using BMA reduces the computational cost of the embedding and extracting process because of its few equations.

The proposed method is divided into two stages: watermark embedding and watermark extracting.

\section{A. WATERMARK EMBEDDING}

This process consists of three steps, in the first step the host image is split into two parts, then the MBA is applied on each

part to select the best locations for concealment by relying on the fitness value of those locations. The size of the cover image and watermark image should be $(512 * 512)$ pixel and $(64 * 64)$ pixel respectively, after that the values of those locations are converted into binary form. In the second step, after splitting the watermark image into two parts and converting it into binary form, these two parts will be encrypted by using improved honey algorithm, the address values of those locations chosen by MBA are used as a key in the encryption process. In the last step, the first part of watermark image will be hidden in the first part of the host image of the best locations chosen by BMA by using least significant bit (LSB), and so on for the second part. The steps of embedding process are shown in algorithm (3), Fig. 2 shows the block diagram of embedding process.

\section{Algorithm 3. Steps of embedding process}

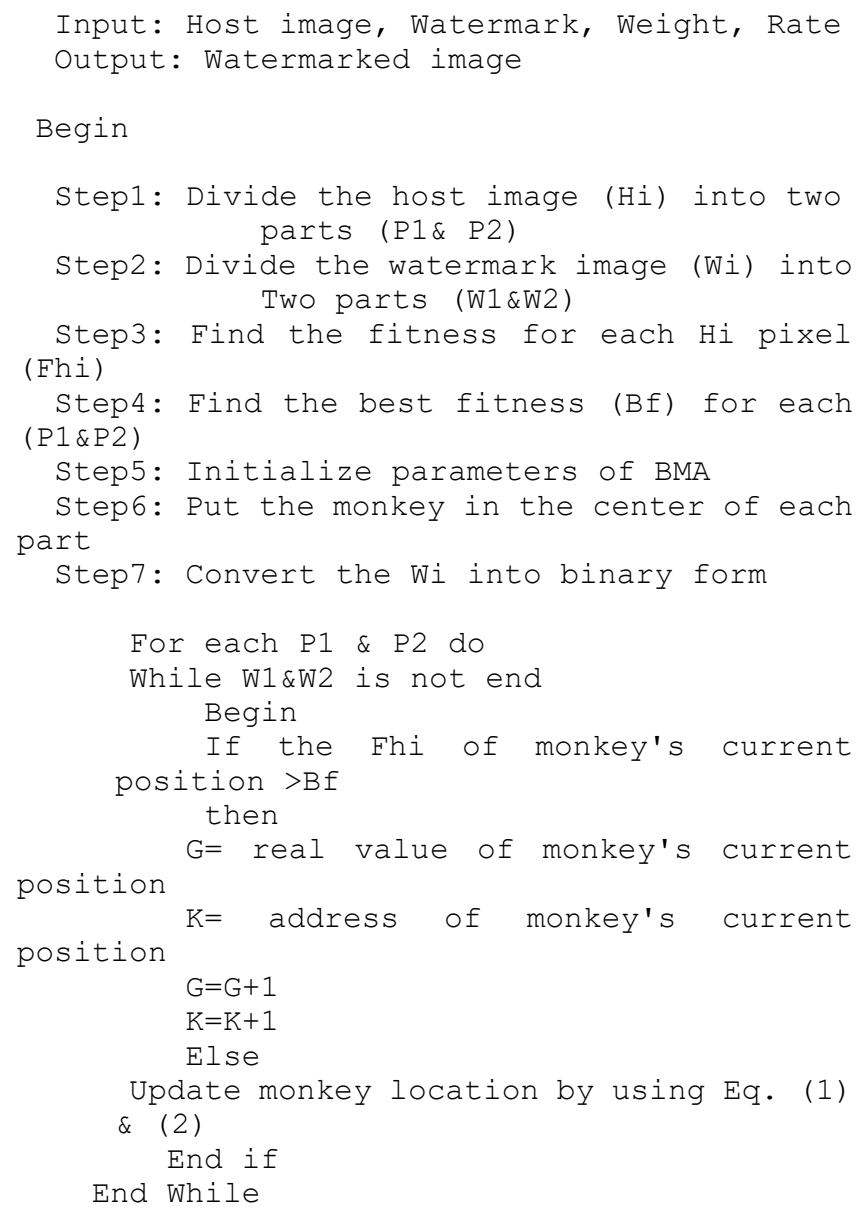




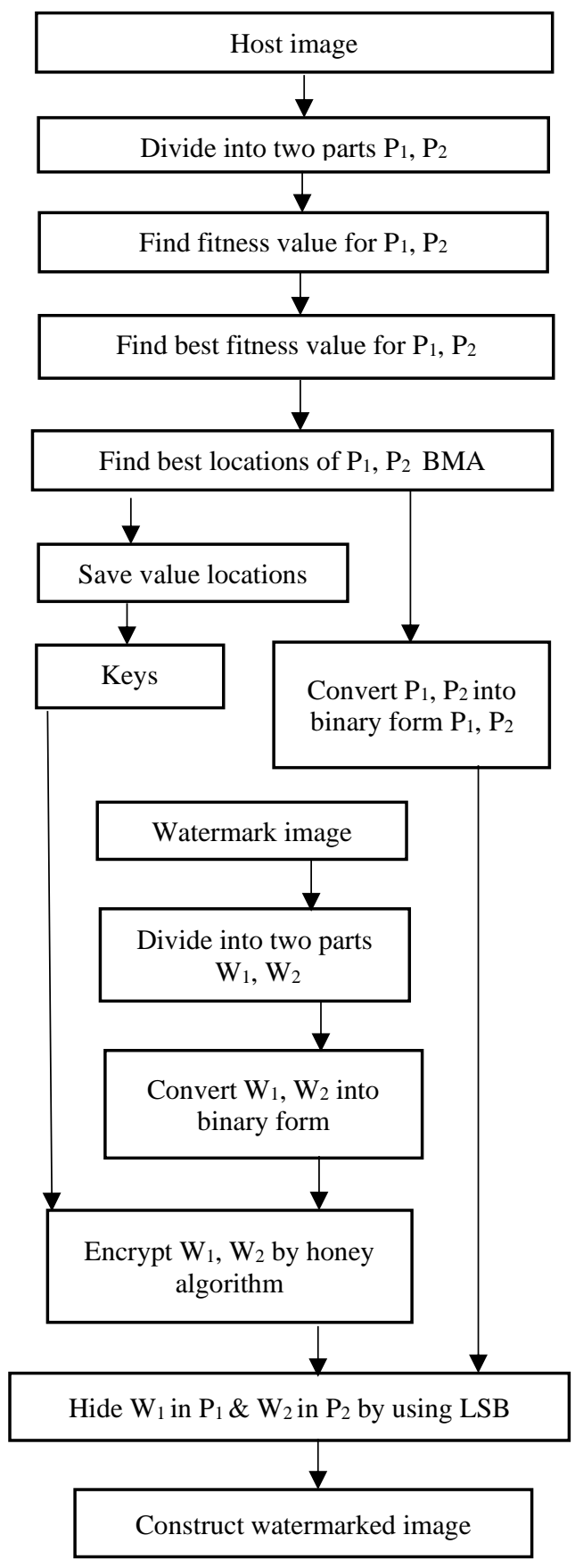

Figure 2. Block diagram of embedding process

\section{B. WATERMARK EXTRACTING}

When the watermarked image is transmitted to the receiver, the receiver extracts the digital watermark by reversing the embedding process. After splitting the watermarked image into two parts, the BMA is applied to identify the location that contains the digital watermark. Moreover, through the use of LSB technique, the encrypted watermark is extracted and decrypted by using honey algorithm that in turn uses the value of locations chosen by BMA as its key. The steps of extraction are shown in algorithm (4), the block diagram of the extraction process is shown in Fig. 3 .

\section{Algorithm 4. Steps of Extracting Process}
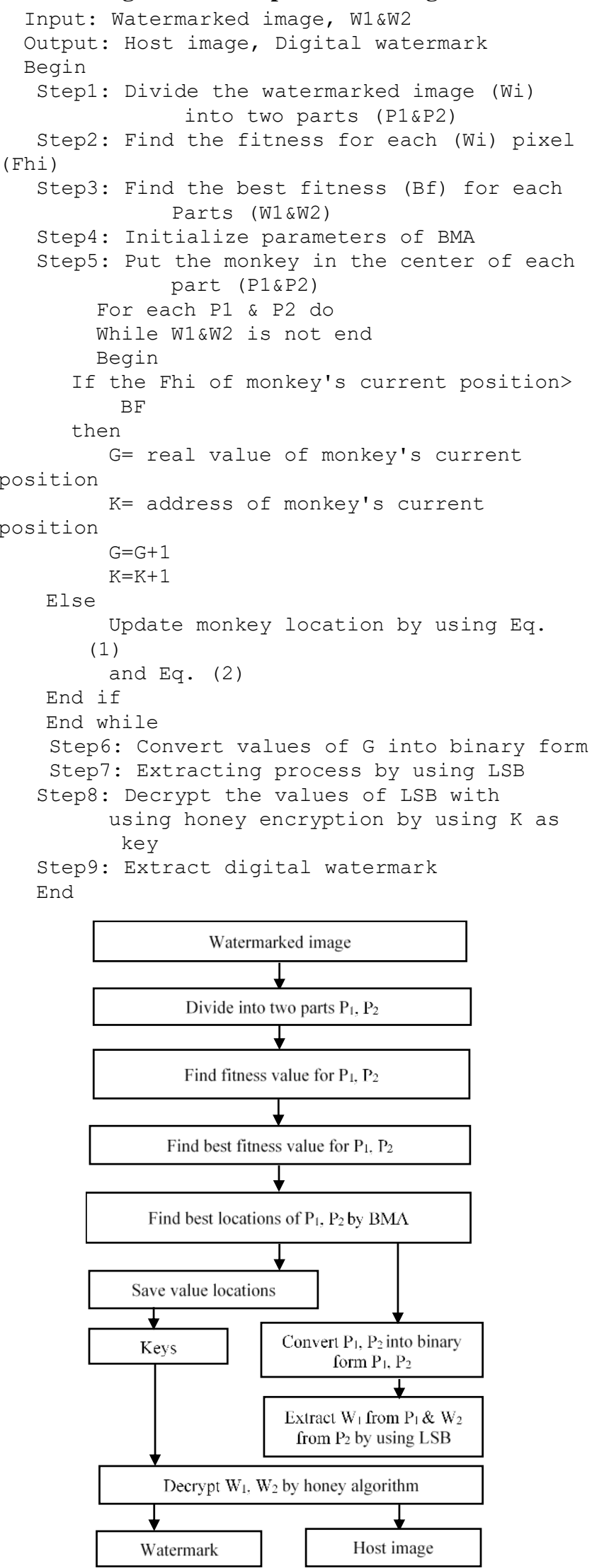

Figure 3. Block diagram of the extraction process 


\section{EXPERIMENTAL RESULT}

The perfect watermarking can be obtained from extracting digital watermark out of watermarked image which is similar to the original watermark before embedding. The main purposes behind this proposed method are concealing a digital watermark with a high security, robustness and maintaining the quality of the image at the same time. This section presents the experiments, the subjective and objective outcomes of the designed method.

Fig. 4 shows the three cover images that will be used in the experiments with the size of $512 * 512$ pixels.

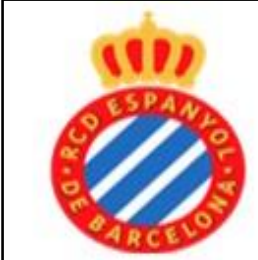

Cover image (a)

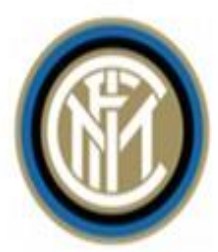

cover Image (b)

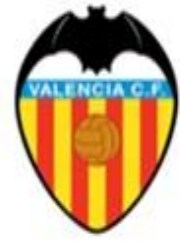

Coverimage (c)
Figure 4. Cover images

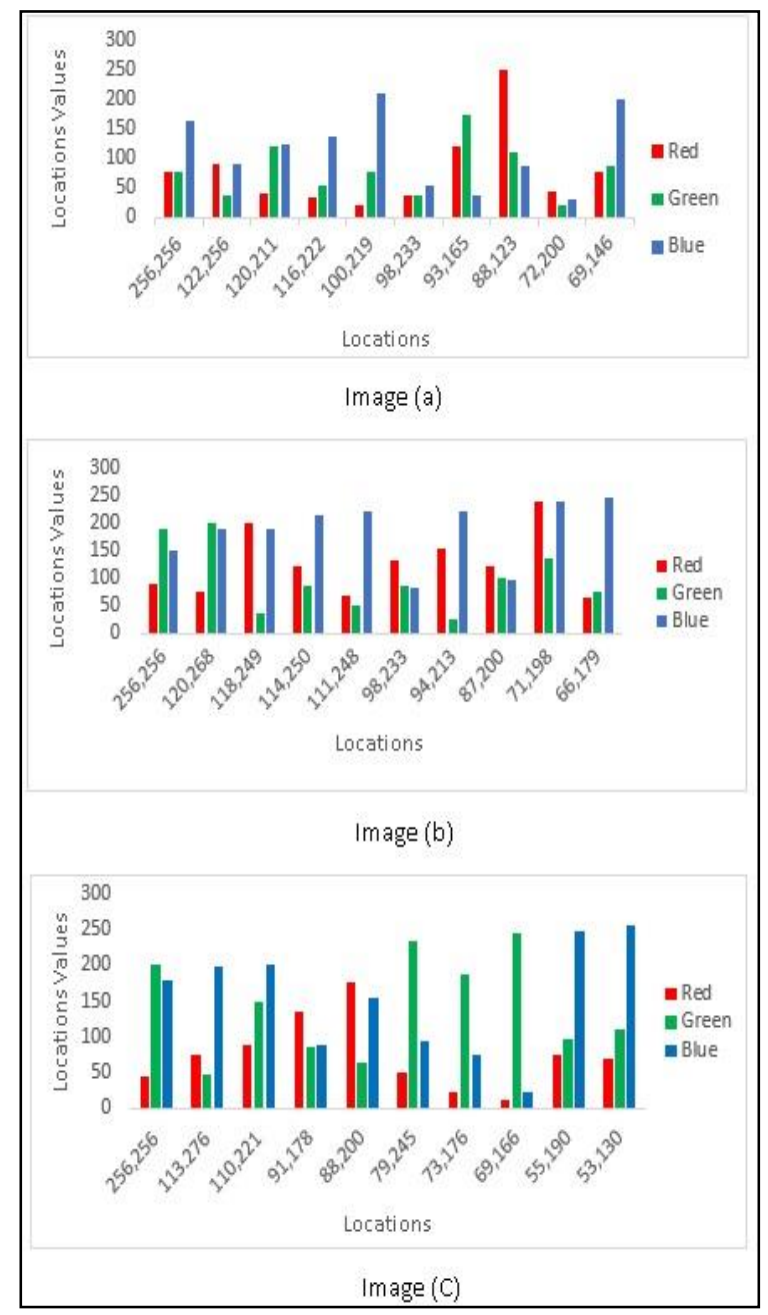

Figure 5. Position and its pixel values that are selected by BMA for all cover image
Positions chosen by the BMA are random (not serial) and are selected by intelligent method. Table 1 and Fig. 5 show some positions with their pixel values that are selected by BMA for image (a), image (b) and image (c).

The digital watermarks with the size of $(64 * 64)$ pixels before and after the encryption process by using honey encryption algorithm are illustrated in Fig. 6. Fig. 7 shows the host image and watermarked image.

Table 2 shows (PSNR) and (MSE) values for host and watermarked image, while Table 3 shows the watermarked images attacked by several attackers such as salt \& pepper noise, Gaussian noise and rotation. It also shows the digital watermark image after its extraction from watermarked images attack.

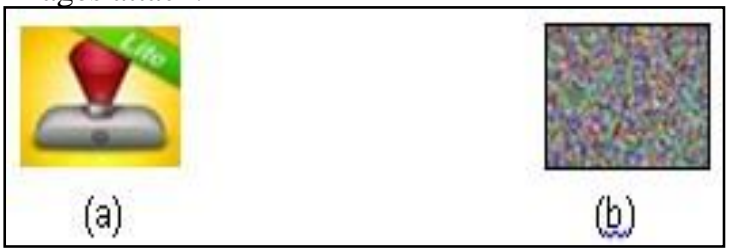

Figure 6. Digital watermark image: (a) before encryption, (b) after encryption

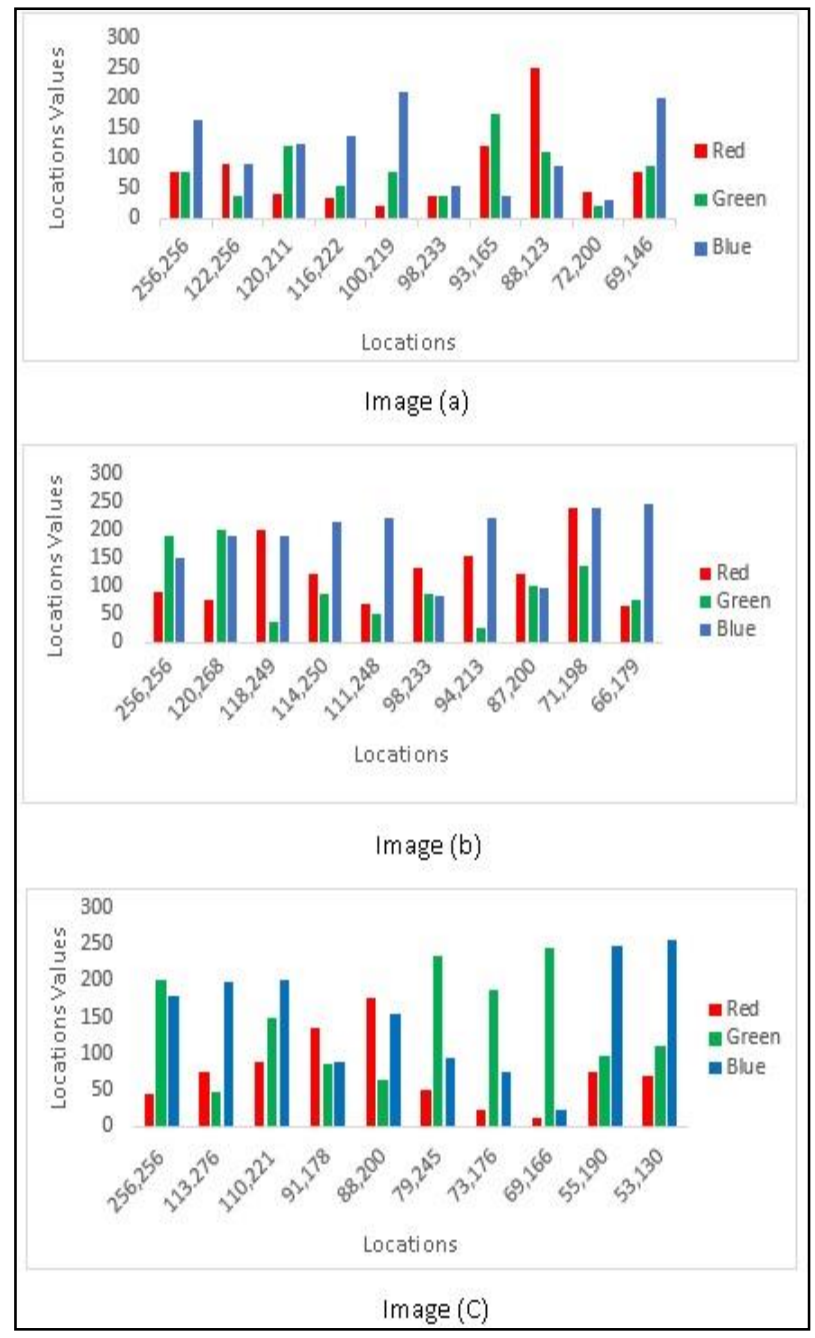

Figure 7. Host and watermarked images 
Table 1. Locations selected by BMA with its values for image (a), image (b) and image (c)

\begin{tabular}{|c|c|c|c|c|}
\hline \multirow[t]{2}{*}{$\mathrm{S}$} & \multicolumn{4}{|c|}{ Image (a) } \\
\hline & Locations & Red value & Green Value & Blue Value \\
\hline 1 & 256.256 & 150 & 189 & 89 \\
\hline 2 & 270.268 & 188 & 200 & 76 \\
\hline 3 & 279.249 & 189 & 36 & 200 \\
\hline 4 & 279.250 & 213 & 86 & 123 \\
\hline 5 & 255.248 & 219 & 49 & 69 \\
\hline 6 & 220.233 & 82 & 87 & 132 \\
\hline 7 & 213.213 & 222 & 25 & 154 \\
\hline 8 & 210.200 & 95 & 100 & 120 \\
\hline 9 & 199.198 & 239 & 137 & 240 \\
\hline 10 & 189.179 & 245 & 76 & 65 \\
\hline \multirow[t]{2}{*}{$\mathrm{S}$} & \multicolumn{4}{|c|}{ Image (b) } \\
\hline & Locations & Red value & Green Value & Blue Value \\
\hline 1 & 256.256 & 178 & 200 & 46 \\
\hline 2 & 256.276 & 199 & 47 & 76 \\
\hline 3 & 243.221 & 200 & 149 & 89 \\
\hline 4 & 199.178 & 88 & 87 & 134 \\
\hline 5 & 185.200 & 153 & 63 & 176 \\
\hline 6 & 180.245 & 93 & 234 & 49 \\
\hline 7 & 177.176 & 76 & 187 & 22 \\
\hline 8 & 172.166 & 22 & 245 & 11 \\
\hline 9 & 169.190 & 247 & 98 & 76 \\
\hline 10 & 158.130 & 255 & 111 & 70 \\
\hline \multirow[t]{2}{*}{$\mathrm{S}$} & \multicolumn{4}{|c|}{ Image (c) } \\
\hline & Locations & Red value & Green Value & Blue Value \\
\hline 1 & 256.256 & 165 & 76 & 76 \\
\hline 2 & 244.256 & 90 & 37 & 89 \\
\hline 3 & 234.211 & 123 & 120 & 40 \\
\hline 4 & 231.222 & 136 & 53 & 34 \\
\hline 5 & 210.219 & 211 & 78 & 22 \\
\hline 6 & 212.233 & 54 & 39 & 39 \\
\hline 7 & 198.165 & 39 & 172 & 120 \\
\hline 8 & 187.123 & 87 & 111 & 249 \\
\hline 9 & 169.200 & 30 & 21 & 45 \\
\hline 10 & 158.146 & 200 & 87 & 76 \\
\hline
\end{tabular}

Table 2. Values of PSNR and MSE of host and watermarked image

\begin{tabular}{|c|c|c|}
\hline Image (512*512) & MSE & PSNR \\
\hline A & 0.00269 & 70.543 \\
\hline B & 0.00278 & 73.106 \\
\hline C & 0.00271 & 71.059 \\
\hline
\end{tabular}

Table 3. Several attacks on watermarked image

\begin{tabular}{|c|c|c|c|}
\hline Attack & Image (a) & Image (b) & Image (c) \\
\hline \multicolumn{4}{|l|}{$\begin{array}{c}\text { Gaussian } \\
\text { noise }\end{array}$} \\
\hline \multicolumn{4}{|l|}{ Rotate $45^{\circ}$} \\
\hline \multicolumn{4}{|l|}{$\begin{array}{c}\text { Salt \& } \\
\text { pepper noise }\end{array}$} \\
\hline \multirow[b]{2}{*}{$\begin{array}{c}\text { Extract } \\
\text { watermark }\end{array}$} & Gaussian noise & Rotate $45^{\circ}$ & Salt \& pepper noise \\
\hline & & & \\
\hline
\end{tabular}


$\mathrm{NC}$ is one of the best methods to evaluate the degree of closeness between the two functions. This measure can be used to determine the extent to which the original image and stego image remain close to each other, even after embedding the data. $\mathrm{NC}$ is calculated between the extracted watermarked and original watermark. Table 4 and Fig. 8 show a comparison between the proposed method and the counterparts [7], [20] and [21].

Table 4. Comparison between the proposed method and other methods against many attacks

\begin{tabular}{|c|c|c|c|c|c|c|c|c|}
\hline \multirow{2}{*}{ Attack } & \multicolumn{2}{|c|}{ Proposed Method } & \multicolumn{2}{|c|}{ M. Saqib et al [7] } & \multicolumn{2}{c|}{$\begin{array}{c}\text { A. Saboori et al } \\
{[20]}\end{array}$} & \multicolumn{2}{|c|}{$\begin{array}{c}\text { S. A et al } \\
{[21]}\end{array}$} \\
\cline { 2 - 10 } & PSNR & NC & PSNR & NC & PSNR & NC & PSNR & NC \\
\hline Gaussian noise & 48.98 & 0.92 & 39.28 & 0.73 & 29.27 & 0.89 & - & 0.88 \\
\hline Rotate 45 & 48.10 & 0.89 & 10.43 & 0.48 & 23.16 & 0.31 & - & 0.75 \\
\hline Salt \& pepper noise & 52.21 & 0.93 & - & 0.69 & 25.14 & 0.74 & - & 0.96 \\
\hline Sharpen & 59.01 & 0.97 & - & - & 26.64 & 0.83 & - & - \\
\hline Median (3*3) filter & 61.76 & 0.98 & 35.21 & 0.65 & 35.47 & 0.97 & - & 0.94 \\
\hline Average (3*3) filter & 68.98 & 1 & - & 0.82 & 32.31 & 0.86 & - & 0.89 \\
\hline JPEG compression & 72.99 & 1 & - & 1 & 36.90 & 0.98 & - & 0.97 \\
\hline Crop & 47.34 & 0.89 & 12.90 & 0.62 & 15.1 & 0.78 & & 0.88 \\
\hline Without attack & 73.10 & 1 & 42.39 & 1 & 40.1 & 1 & 49.65 & 1 \\
\hline
\end{tabular}

The NC values for the proposed system obtained higher results compared to the $\mathrm{NC}$ values in the previous works, where it is very close to the ideal value which is 1 . Table 5 shows the comparison of the proposed method and other methods in term of availability, reliability, and confidentiality. Therefore, the proposed method is more robust and secured than [7], [20] and [21].

Table 5. Comparisons of the proposed method with other methods

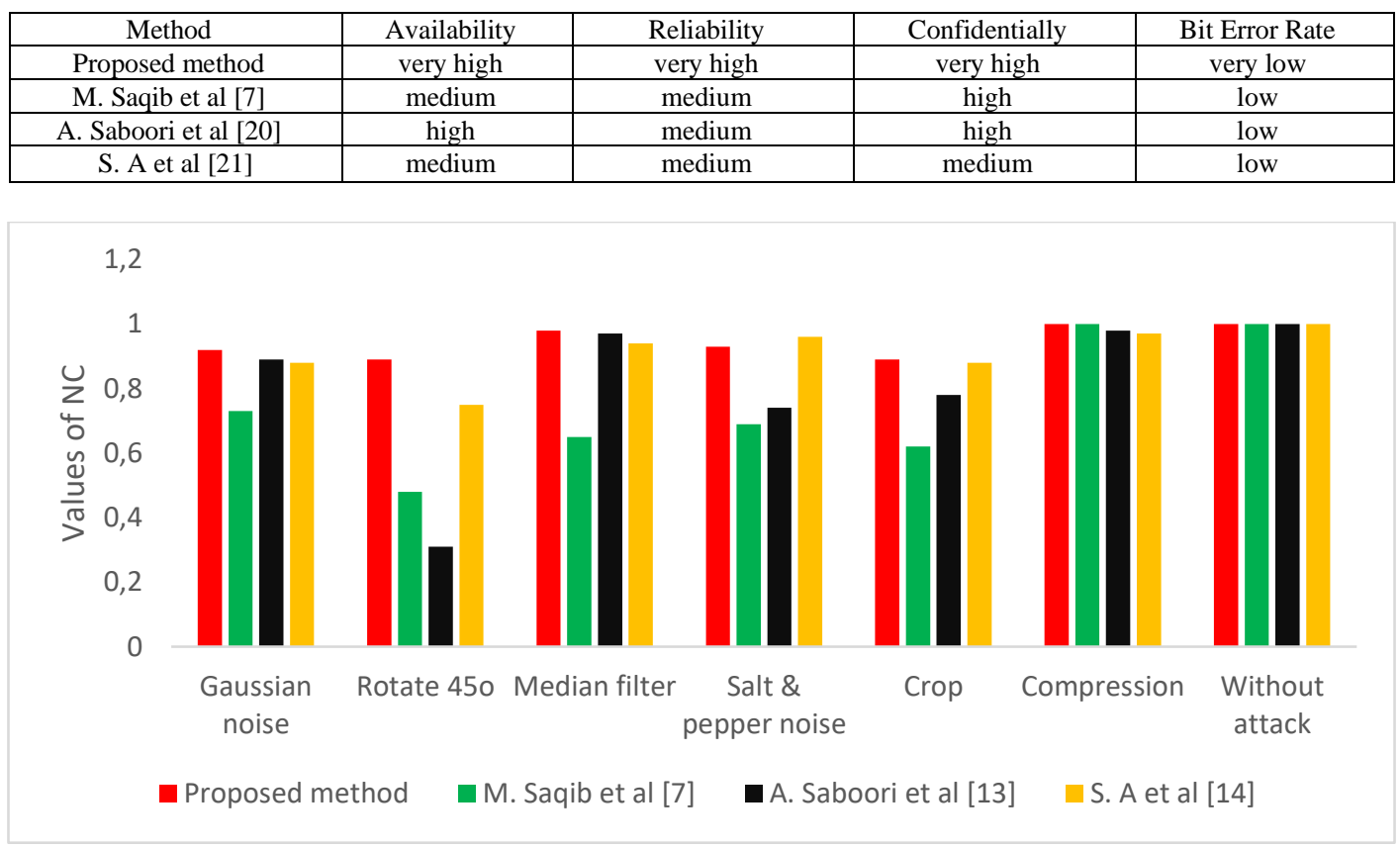

Figure 8. Comparison of NC between the proposed method and the other methods

\section{CONCLUSION}

The current paper presents a new scheme depending on the two important algorithms that are the BMA and improved honey encryption. The outcomes of this paper confirmed the following:

- This method can solve the problem of the security through using honey algorithm.

- Increase the security by using the address of locations selected by BMA as keys for the honey algorithm
- Limits the attacker's ability to remove the digital watermark as the location that is used to hide the digital watermark is selected randomly in an intelligent way by using the BMA, as shown in Table 1 and Fig. 5. And this point indicates the effectiveness of the proposed system.

- The value of PSNR is high while the value of MES is low as shown in Table 2. This indicates the imperceptibility of the proposed system. 
- As seen in Table 3, the proposed method provided the robustness through withstanding against many attacks.

- Because of the speed of the BMA and improved honey algorithm, the time consumed for the embedding and extraction process is little.

\section{References}

[1] M. Begum and M. S. Uddin, "Digital image watermarking techniques: a review," MDPI Information Journal, vol. 11, no. 110, pp. 2-42, 2020. https://doi.org/10.3390/info11020110.

[2] H. Tao, L. Chongmin, J.M. Zain, A.N. Abdalla, "Robust image watermarking theories and techniques: a review," J. Appl. Res. Technol. vol. 12, issue 1, pp. 122-138, 2014. https://doi.org/10.1016/S1665-6423(14)71612-8.

[3] Y. Zhang, "Digital watermarking technology: a review," Proceedings of the ETP International Conference on Future Computer and Communication, Wuhan, China, 6-7 June 2009, pp. 250-252. ISBN: 978-0-7695-3676-7, https://doi.org/10.1109/FCC.2009.76.

[4] I. Cox, M. Miller, J. Bloom, J. Fridrich, T. Kalker, Digital Watermarking and Steganography, 2nd ed., Morgan Kaufmann Series in Multimedia Information and Systems: Burlington, Massachusetts, 2007, 624 p. eBook ISBN: 9780080555805.

[5] T. Minamoto and K. Aoki, "A blind digital image watermarking method using interval wavelet decomposition," International Journal of Signal Processing, Image Processing and Pattern Recognition, vol. 3, no. 2, pp. 59-72, 2010.

[6] Q. Ying and J. Lin, "Robust digital watermarking for color images in combined DFT and DT-CWT domains," Mathematical Biosciences and Engineering, vol. 16, issue 5, pp. 4788-4801, 2019. https://doi.org/10.3934/mbe.2019241.

[7] M. Saqib and S. Naaz, "An improvement in digital image watermarking scheme based on singular value decomposition and wavelet transform," Asian Journal of Computer Science and Technology, vol. 8, no.1, pp. 62-68, 2019.

[8] S. Kaur and H. Jindal, "Enhanced image watermarking technique using wavelets and interpolation," I.J. Image, Graphics and Signal Processing, vol. 7, pp. 23-35, 2017. [Online]. Available at: http://www.mecs-press.org/. https://doi.org/10.5815/ijigsp.2017.07.03.

[9] T. S. Fun, A Samsudin and Z. F. Zaaba, "Enhanced security for public cloud storage with honey encryption," American Scientific Publishers, pp. 1-5, 2015.

[10] N. S. Noorunnisa and K. R. Afreen, "Review on honey encryption technique," International Journal of Science and Research (IJSR), vol. 5, issue 2, pp. 1683-1686, 2016. https://doi.org/10.21275/v5i2.NOV161555.

[11] S. Mirjalili, A. H. Gandomi, S. Z. Mirjalili, S. Saremi, H. Faris, and S. M. Mirjalili, "Salp swarm algorithm: a bio-inspired optimizer for engineering design problems," Adv. Eng. Softw, vol. 114, pp. 163-191, 2017. https://doi.org/10.1016/j.advengsoft.2017.07.002.

[12] M. Mahmood and B. Al-Khateeb, "The blue monkey: A new nature inspired metaheuristic optimization algorithm," Periodicals of Engineering and Natural Sciences, vol. 7, no. 3, pp. 1054-1066, 2019. https://doi.org/10.21533/pen.v7i3.621.
[13] S. Bekkouche \& A. Chouarfia, "A new watermarking approach based on combination of reversible watermarking and CDMA in spatial and DWT domain," International Journal of Security (IJS), vol. 5, issue 1, pp. 1-12, 2011. https://doi.org/10.1117/12.896098.

[14] N. Jain, R. Gupta, "A novel approach for digital image watermarking using cryptography," International Journal on Recent and Innovation Trends in Computing and Communication, vol. 2, issue 9, pp. 29082913, 2014.

[15] D. G. Savakar, S. Pujar, "Digital image watermarking at different levels of DWT using RGB channels," International Journal of Recent Technology and Engineering, vol. 8, issue 5, pp. 599-570, 2020. https://doi.org/10.35940/ijrte.D6821.018520.

[16] A. E. El Hossaini, M. El Aroussi, K. Jamali, S. Mbarki, and M. Wahbi, "A new robust blind watermarking scheme based on steerable pyramid and DCT using Pearson product moment correlation," Journal of Computers, vol. 9, no. 10, pp. 2315-2327, 2014. https://doi.org/10.4304/jep.9.10.2315-2327.

[17] M. A. Abdullah, S. S. Dlay, and W. L. Woo, "Securing iris images with a robust watermarking algorithm based on discrete cosine transform," Proceedings of the 10th International Conference on Computer Vision Theory and Applications, Berlin, Germany, 2015, pp. 108-114. https://doi.org/10.5220/0005305701080114.

[18] H. Prasetyo and C.-H. Hsia, "Lossless progressive secret sharing for grayscale and color images," Multimedia Tools and Applications, vol. 78, pp. 24837-24862, 2019. https://doi.org/10.1007/s11042-0197710-5.

[19] S. Malik and R. K. Reddlapalli, "Histogram and entropy based digital image watermarking scheme," International Journal of Information Technology, vol. 11, issue 2, pp. 373-379, 2019. https://doi.org/10.1007/s41870-018-0259-0.

[20] A. Saboori and S. A. Hosseini, "A new method for digital watermarking based on combination of DCT and PCA," Proceedings of the Conference: Telecommunications Forum Telfor (TELFOR), Belgrade, 2014, pp. 1-4. https://doi.org/10.1109/TELFOR.2014.7034461.

[21] S. A. Ali, M. J. Jawad, and M. A. Naser, "Copyright protection for digital image by watermarking technique," Journal of Information Processing Systems, vol. 13, no. 3, pp. 599-617, 2017. https://doi.org/10.3745/JIPS.03.0074.

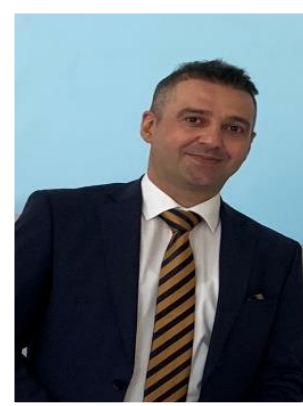

OMAR YOUNIS has PhD in computer science at Garmian University. He is an Assistant Prof. at College of Science, University of Garmain, region of Kurdistan, Iraq. His research interests include image processing, biometrics, pattern recognition, Steganography, A.I, computer networks, software. 